\section{Vol. 69, No. 50}

In the report "The Advisory Committee on Immunization Practices' Interim Recommendation for Use of PfizerBioNTech COVID-19 Vaccine - United States, December 2020," on page 1922, in the third paragraph, the third and fourth sentences should have read Consistent high efficacy $(\geq 92 \%)$ was observed across age, sex, race, and ethnicity categories and among persons with underlying medical conditions. Efficacy was similarly high in a secondary analysis including participants both with or without evidence of previous SARS-CoV-2 infection. Although numbers of observed hospitalizations and deaths were low, the available data were consistent with reduced risk for these severe outcomes among vaccinated persons compared with that among placebo recipients.

\section{Vol. 70, No. 3}

In the report "Evaluation of Abbott BinaxNOW Rapid Antigen Test for SARS-CoV-2 Infection at Two CommunityBased Testing Sites - Pima County, Arizona, November 3-17, 2020," on page 103, in Table 2 , the positive predictive value (PPV) and 95\% confidence interval (95\% CI) for the BinaxNOW antigen test performance for asymptomatic participants should have read "91.7 (80.0-97.7)." Also on that page, the second sentence of the fourth full paragraph should have read "Community testing strategies focused on preventing transmission using antigen testing should consider serial testing (e.g., in kindergarten through grade 12 schools, institutions of higher education, or congregate housing settings), which might improve test sensitivity in detecting infection (10)." 